\title{
Resultados de 3 años de experiencia en reproducción asistida en el centro de biomedicina reproductiva - FECUNDAR - Cali, Colombia
}

\author{
Jaime Saavedra S.*; Rodrigo Muñoz Gil**; Marco Julio Velásquez***; Consuelo Jaramillo****
}

\section{RESUMEN}

En este trabajo se reporta los resultados en reproducción asistida (FIV) del Centro de Biomedicina Reproductiva (FECUNDAR) Cali - Colombia durante el período 1995-1998.

Se incluyen un total de 254 ciclos iniciados dando como resultado 55 embarazos clínicos con 38 nacidos vivos, lo que da una tasa del $20.2 \%$ de nacidos vivos pos trasferencia; se utilizaron dos esquemas de inducción de ovulación : GnRH más hMG y Citrato de Clomifeno más hMG, las tasas de embarazo fueron $28.1 \%$ y $6.7 \%$ respectivamente. El promedio de edad de las pacientes fue de 35 años, encontrándose la mayor tasa de embarazos entre los 25 y 35 años $(25.5 \%$ ) cayendo dramáticamente a $9 \%$ después de los 40 años. La principales causas de ingreso al programa de FIV fue la patología tubárica, 120 casos, y Otras causas femeninas 48 casos con unas tasas de embarazos del $32.3 \%$ y $34.3 \%$ respectivamente, la infertilidad masculina 19 casos, la múltiple 40 casos, la inexplicada 27 casos presentaron tasas de embarazos de $15.4 \%$, $17.5 \%$ y $13 \%$ respectivamente.

PALABRAS CLAVES: Inducción de ovulación, recuperación de ovocitos, transferencia embrionaria.

\section{SUMMARY}

In this work, the results of assisted reproduction (FIV) are presented from the Centro of Biomedicine Reproductive (FECUNDAR) Cali - Colombia during the years 1995-1998.

There are included a total of 254 cycles giving as a result 55 clinic pregnancies with 38 living born childs, these give us a rate after transfer of $20.2 \%$ of living born childs; There were used two types of schemes of ovulation induction: GnRh plus hMG and Clomiphene Citrate plus hMG, the rates of pregnancy were $28.1 \%$ and $6.7 \%$. The average of age from the patiens were 35 years, founding the highest rate of pregnancy between 25 and 35 years $(25.5 \%)$ bringing dramatically these down to a $9 \%$ after 40 years. The main reasons for joining the FIV program was the tubal pathology 120 cases, and other feminine causes 48 cases, with a rate of pregnancy of $32.3 \%$ and $34.3 \%$ respectively, masculine infertility 19 cases, 40 cases of multiple causes, and unexplainable 27 cases, these motives, displayed a rate of pregnancy of $15.4 \%, 17.5 \%$, and $13 \%$ respectively.

KEY WORDS: Ovulation induction, oocyte colletion, embryo transfer.

Los primeros embarazos y nacimientos exitosos por fertilización in vitro (FIV) fueron reportados por Steptoe y Edwards en 1978 (1) y por Lopata en 1980 (2). Estos resultados fueron la culminación de esfuerzos científicos realizados por más de una centuria.

Walter Heape (3) en 1981 demostró la posibilidad de recobrar embriones en estado de preimplantación mediante el lavado del oviducto de coneja y transferir los embriones a una coneja receptora continuando su normal

\footnotetext{
* Profesor Titular. Departamento de Obstetricia y Ginecología Universidad del Valle. Jefe del Servicio de Medicina Reproductiva Departamento de Obstetricia y Ginecología. Universidad del Valle. Director General FECUNDAR.

** Obstetra y Ginecólogo. Biomedicina Reproductiva. Director Médico FECUNDAR.

Obstetra y Ginecólogo. Biomedicina Reproductiva. FECUNDAR. Bióloga. FECUNDAR.
}

desarrollo. El trabajo pionero de Heape y el éxito en la trasferencia de embriones en otras especies, hizo que el interés científico se enfocara al cultivo de embriones en el laboratorio. En 1949 Hammond (4) desarrolló un medio de cultivo complejo que permitía el crecimiento de embriones de ratas de ocho células a blastocisto y Whitten (5) demostró en 1956 que un medio de cultivo químicamente definido podría ser igualmente efectivo. En 1952 Austin (6) y Chang (7) descubrieron la capacitación espermática con técnicas simples de cultivo y en 1959 Chang (8) reportó la primera fertilización in vitro de óvulos de conejas.

El nacimiento de Louise Brown en 1978, el primer bebé obtenido por una fertilización in vitro (FIV) y trasferencia embrionaria (TE), disparó una sucesión de desarrollos médicos, biológicos y técnicos que han simplificado el procedimiento aumentando su eficiencia y expandido sus indicaciones. A partir de 1980 la FIV-TE 
pasó de ser un procedimiento experimental a ser parte integral del manejo en la medicina reproductiva, afectando dramáticamente el manejo tradicional de los problemas de infertilidad.

\section{Materiales y métodos}

\section{Pacientes}

De diciembre de 1995 a diciembre de 1998, fueron atendidos en el Centro de Biomedicina Reproductiva FECUNDAR, parejas con diferentes problemas en su fertilidad; de éstas, 254 ingresaron al programa de fertilización in vitro (FIV) y trasferencia embrionaria (TE). Del número anterior 120 presentaba patología tubárica, 48 presentaban otras causas femeninas de infertilidad (endometriosis, adherencias pélvicas, alteraciones endocrinas, alteraciones cromosómicas), 40 presentaban patología múltiple, 19 tenían un compromiso severo del factor masculino y 27 de estas parejas presentaban una infertilidad de causa desconocida.

La edad de las mujeres variaba entre 20 y 45 años con un promedio de 35 años.

Los requerimientos mínimos de las pacientes para entrar al programa de FIV son tener una cavidad uterina normal, una fuente adecuada de ovocitos, suficientes espermatozoides para llevar acabo la fertilización, ya sea por inseminación rutinaria o por técnicás de fertilización asistida.

\section{Protocolos de inducción de ovulación para FIV-TE}

\section{Citrato de Clomifeno - hMG}

En 23 pacientes se hizo inducción de la ovulación con citrato de clomifeno (CC) $50 \mathrm{mg} /$ diarios por cinco días iniciando el segundo día del ciclo menstrual, adicionando gonadotropina menopáusica humana, hMG (Pergonal, del laboratorio Serono) 300 IU diarias del 2 al 6 y del día 7 al 8 la dosis de hMG se ajustaba individualmente de acuerdo a los niveles diarios de estradiol (E2) y a las medidas del crecimiento folicular determinado por ultrasonido del día 6 al 9 del ciclo (9-13)

$\mathrm{Si}$ el aumento de los estrógenos es constante y no excesivo se continúa con la misma dosis hasta que los niveles de $E_{2}$ alcancen entre $800-2500 \mathrm{pg} / \mathrm{mL}$. Si en ambos ovarios el número total de estructuras foliculares medidas por ultrasonografía no excede de 10 , y teniendo más de cuatro folículos con un diámetro superior a 18 $\mathrm{mm}$, se administra la dosis ovulatoria de gonadotropina corionica humana (hCG) en cantidad de 5000 UI el día 9 del ciclo, y 36 horas después de la aplicación de esta hormona se realiza la aspiración de todos folículos accesibles con el objeto de colectar tantos ovocitos como fuera posible mediante ecografía trasvaginal.

\section{Agonistas de GnRH y h MG}

En la inducción de ovulación con los análogos de GnRh más gonadotropinas usamos los esquemas ya establecidos de protocolo largo y corto (14-15) (Fig. 1).

Protocolo largo. En el protocolo largo el tratamiento con gonadotropinas se inicia únicamente después de tener una supresión adecuada de la hipófisis. La desensi- bilización de la hipófisis se produce con la administración del agonista de GnRH, éste se puede iniciar a mitad de la fase lútea (día 21) del ciclo precedente o en la fase folicular temprana (día 2 de la menstruación) del ciclo presente (16-18). Típicamente la menstruación suele aparecer entre 5 y 10 días después de iniciar el análogo en la fase lútea, al cabo de los cuales la determinación de estradiol y la ultrasonografía deben ser utilizadas para confirmar la adecuada supresión y la ausencia de formación de quistes ováricos. La desensibilización se confirma cuando los niveles de estradiol son menores de $40 \mathrm{pg}$ / $\mathrm{m}$.

El agonista se puede administrar en presentación de depósito, inyecciones diarias subcutáneas o en nebulizaciones nasales y debe ser continuado concomitantemente con los inductores de ovulación hasta el día de la administración de la hCG.

Este protocolo fue aplicado en 50 pacientes utilizando Acetato de Leuprolide de depósito (Laboratorios Abbot) $3,75 \mathrm{mg} / \mathrm{IM}$, en fase lútea y en 133 pacientes igual dosis y presentación en fase folicular; en 21 pacientes utilizamos en fase lútea la presentación subcutánea. En dosis de $1 \mathrm{mg} /$ diario;

Protocolo corto. El protocolo corto (llamado flare up por los ingleses) incorpora el efecto agonista de los análogos de GnRH y la estimulación de la FSH/LH endógenas secretadas por la hipófisis (19-22). Los análogos de la GnRh se inician el 1 ó 2 día de la menstruación y las gonadotropinas se inician igualmente el 2 ó 3 día del ciclo.

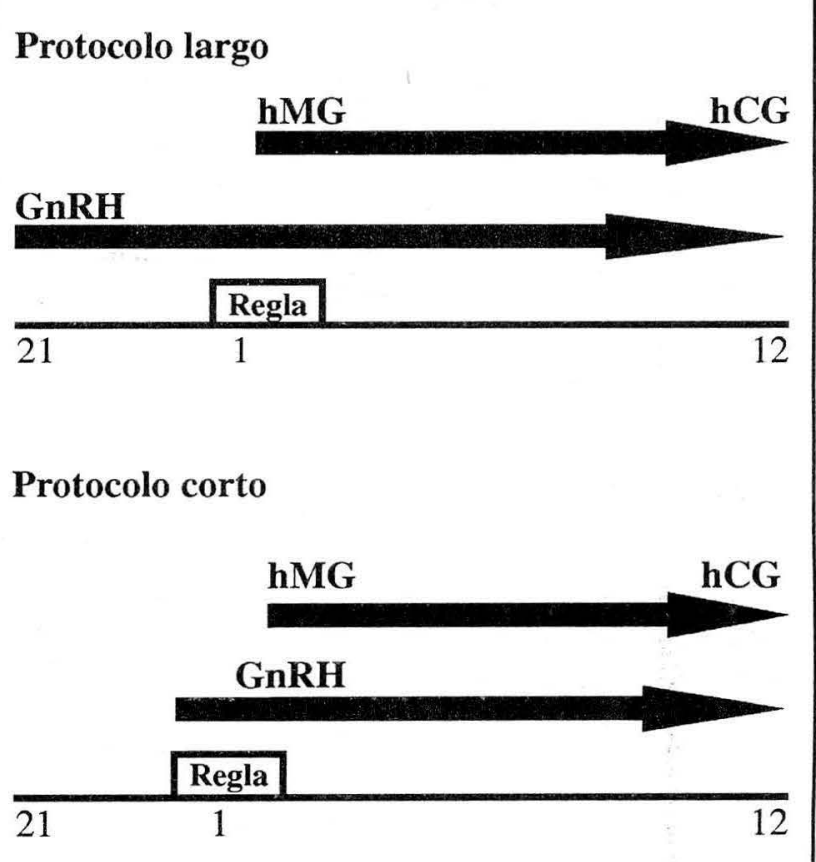

Figura $0023 \mathrm{i} 01$

REPRESENTACION ESQUEMATICA DE DOS TRATAMIENTOS PARA INDUCCION DE OVULACION CON ANALOGOS DE GNRH 
Como en el protocolo largo, el agonista se suspende cuando la hCG es administrada.

Este protocolo fue aplicado a 15 pacientes utilizando Acetato de Leuprolide subcutáneo en dosis de $1 \mathrm{mg} / \mathrm{d}$.

En ambos protocolos la dosis de gonadotropina menopáusica utilizada fue en promedio $300 \mathrm{UI} /$ diarias de Pergonal (Laboratorio Serono). El día de la aplicación de gonadotropina corionica humana (HCG) depende del tamaño folicular y el nivel de estradiol; nuestra unidad tiene el siguiente criterio para aplicar la HCG en FIV: mínimo cuatro folículos de $18 \mathrm{~mm}$ de diámetro y un nivel de estradiol mínimo $800 \mathrm{pg} / \mathrm{mL}$ y un máximo $3000 \mathrm{pg} /$ $\mathrm{mL}$. Como dosis ovulatoria de HCG utilizamos $10.000 \mathrm{U}$.

\section{Recuperación de ovocitos y valoración de la madu- ración ovocitaria}

La recuperación de ovocitos para FIV se realiza entre 32 y 36 horas después de haber inyectado la HCG (23); mediante ecografía trasvaginal, para lo cual se acopla al transductor una aguja No 17 que se fija a un aparato de aspiración automática, el cual mantiene una presión entre 100 y $120 \mathrm{~mm}$ de mercurio (24-29).

Rutinariamente después de aspirar cada folículo se realiza un lavado de éste con $2 \mathrm{~mL}$ de medio de cultivo (Ham F-10) (30).

Para realizar el procedimiento se coloca a la paciente un goteo intravenoso de un agente anestésico de corta acción (Diprivan, Laboratorio Zeneca) teniendo presente que éste no dura mas de 15 minutos (31-34).

Después de la recolección del fluido folicular, el aspirado se coloca rápidamente en una caja de Petri para determinar la presencia de ovocitos. Los ovocitos se reconocen por su apariencia firme y transparente. Si hay presencia de sangre en el cúmulus se lava cuidadosamente ya que ésta afecta negativamente la división (35).

Habitualmente se evalúa la madurez ovocitaria por la expansión y laxitud del cúmulus (36-37); los ovocitos se clasifican rápidamente como maduros cuando presentan el cúmulus expandido y la corona en forma uniformemente radiada. Los ovocitos inmaduros se asocian con una matriz del cúmulus mas compacta que indica un estadio intermedio de madurez, junto a una corona compacta inapreciable.

El cultivo de los ovocitos hasta el momento de la inseminación se lleva acabo en pocillos con 600 uL de medio de cultivo cubiertos con aceite mineral, disponiendo de varios ovocitos juntos.

Preparación del semen e inseminación de los ovocitos

La preparación del semen para fertilización de los ovocitos se realiza mediante la técnica del 'swim up' (38) , con ésta se consigue la selección de los espermatozoides con mejor movilidad dejando que éstos naden hacia la superficie del sobrenadante; quedando en un conglomerado más o menos compacto los espermatozoides inmóviles, móviles sin progresión y otras células presentes en el eyaculado.

La inseminación de los ovocitos se realiza en microgotas colocándolos en las gotas que contienen la concentración adecuada de espermatozoides, la cual es de 20.000 espermatozoides móviles progresivos por ovocito (39-41).
Una vez obtenidos los embriones permanecen en un medio ambiente adecuado para su división sucesiva, para lo cual se mantienen individualmente en microgotas de $20 \mathrm{uL}$ de cultivo cubiertas por aceite mineral y en un ambiente de $\mathrm{CO}_{2}$ del $5 \%$ a una temperatura de 37 grados centígrados.

En el centro se realiza la trasferencia embrionaria, habitualmente, al segundo día de la fertilización cuando los embriones presentan de cuatro a seis blastomeras y usualmente no se transfieren más de tres embriones por paciente.

Como rutina se complementa la fase lútea con $1000 \mathrm{U}$ de HCG los días 1-3-5 pos transferencia, se mide estradiol y progesterona los días 7, 9 y 12 en este último se realiza medición de B-HCG para determinar si la paciente se embarazó.

\section{Resultados}

La tabla 1 muestra los resultados de 3 años de experiencia en reproducción asistida en el Centro de Biomedicina Reproductiva - FECUNDAR de la Ciudad de Santiago de Cali, Colombia.

Para los cálculos estadísticos se consideran como embarazos clínicos todos aquellos que presentaban una elevación adecuada de B-HCG, y con ecografía se mostraba la presencia de un saco gestacional en la cavidad uterina.

De 188 trasferencias obtuvimos 55 embarazos clínicos lo que correspondió a un 29.3\%; 38 terminaron en partos o cesáreas lo cual equivale a una tasa de nacidos vivos del 20,2\%. La tasa de aborto espontáneo en nuestra experiencia fue del $30 \%$, y aunque es más alta que la de la población fértil (36-37) es comparable con la tasa de la población infértil (38).

Tabla 1

CICLOS DE TRATAMIENTO Y EMBARAZO CLINICO PARA FIV 1995-1998

\begin{tabular}{|lr|}
\hline Resultados & FIV \\
\hline Ciclos Iniciados & 254.0 \\
Ciclos discontinuados & 47.0 \\
& \\
Aspiraciones & 207.0 \\
Trasferencias & 188.0 \\
Embarazos clínicos & 55.0 \\
Parto o Cesárea & 38.0 \\
\% de ciclos discontinuados & 18.5 \\
\% de Trasferencias por aspiración & 90.8 \\
\% de Embarazos clínicos por: & 26.6 \\
$\quad$ Aspiración & 29.3 \\
$\quad$ Trasferencia & \\
\% de nacidos vivos por: & 18.4 \\
$\quad$ Aspiración & 20.2 \\
Trasferencia & 69.1 \\
Embarazos clínicos & \\
\hline
\end{tabular}


La tabla 2 presenta la respuesta en número de aspiraciones y embarazos clínicos a los esquemas terapéuticos en los cuales se utilizó un agonista de GnRH (Acetato de Leuprolide) más gonadotropinas; obteniendo en $231 \mathrm{ca}-$ sos una tasa de cancelaciones del $16.9 \%$ y de embarazos clínicos del $28.1 \%$. Cuando se utilizó Citrato de Clomifeno (CC) más gonadotropinas en 23 casos, la tasa de cancelaciones fue del $34.8 \%$ y de embarazos clínicos del $6.7 \%$. No se encontró diferencia estadística en las tasas de embarazos en cuanto a la utilización del agonista de GnRH en forma de depósito o subcutáneo en el protocolo largo para inducción de ovulación; en el trabajo inicial se utilizó la forma de depósito para ciclar grupos de pacientes por costos de laboratorio.

De acuerdo con las causas de infertilidad que determinó el ingreso de los pacientes al programa de FIV/TE; las mejores tasas en cuanto embarazos clínicos se encontraron en pacientes con Factor Tubárico y Otras Causas Femeninas $(32.3 \%$ y $34.3 \%$ ); la causa Masculina, la Múltiple y la Inexplicada presentaron una tasa de embarazo clínico de $15.4 \%, 17.5 \%$ y $13 \%$, respectivamente, tabla 3 .

En la tabla 4 observamos que la tasa de embarazo en el grupo de edad entre los 25 y 35 años fue del $25.7 \%$, esta tasa cae dramáticamente a partir de los 40 años al $9 \%$.

\section{Discusión}

Desde que nació el primer bebé a partir de una fertilización in vitro (FIV) en 1978, la tecnología de la reproducción asistida ha experimentado un desarrollo considerable, particularmente en el ámbito de la inducción de la ovulación y la recuperación de los ovocitos.
Los buenos resultados obtenidos mediante FIV en los casos de enfermedad tubárica llevaron su aplicación en infertilidad inexplicada y en endometriosis (1983) y en la subfertilidad masculina (1985). En 1993 la indicación para casos de infertilidad sólo masculina pasó del $15 \%$ al $21 \%$. La proporción de infertilidades no explicadas permaneció estable (10\%). Los factores estrictamente tubáricos representaron únicamente un $45 \%$ de las indicaciones, aunque otras pacientes con enfermedad tubárica fueron incluidas en factores múltiples (18.4\%) (45).

Desde los inicios de la década de 1980 el número de protocolos para inducción de ovulación en FIV han sido numerosos hasta incluir aquellos que protegen del pico de la LH, así como un gran número de agentes usados para inducción del desarrollo folicular. Estos agentes son Citrato de Clomifeno, hMG, hormona folículo estimulante pura derivada de la orina (u-FSH), FSH recombinante (r-FSH), agonista de la GnRH y hormona de crecimiento $(\mathrm{GH})$ como cotratamiento en la inducción de la ovulación.

Cuando se analizaron los resultados del uso de CChMG, la tasa de embarazo general reportada por el uso de estos agentes fue del rango del $15 \%$ al $17 \%$ por aspiración ó 20\% por trasferencia (46-47). Cuando se compararon los ciclos de hMG versus ciclos de CC-hMG, se encontró lo siguiente: 1) crecimiento rápido, de tal forma que al momento de la aspiración los folículos son más grandes y fáciles de aspirar; 2) una mayor respuesta a la producción de estradiol; 3 ) una mayor elevación en la producción de progesterona; 4 ) un alto porcentaje en la iniciación de la LH; 5) una frecuencia alta de respuesta endocrina pobre o anormal. El pico espontáneo de LH ocurre en el $50 \%$ de los pacientes cuando CC-hMG se inicià el día 2

Tabla 2

NUMERO DE ASPIRACIONES Y EMBARAZOS CLINICOS SEGUN ESQUEMA DE TRATAMIENTO

\begin{tabular}{|lcccccc|}
\hline & No & Cancelación & \% & Asp & E. Clin & \% \\
\hline GnRH + Otro & 231 & 36 & 16 & 192 & 54 & 28.1 \\
Clomifeno + Otro & 23 & 8 & 34.8 & 15 & 1 & 6.7 \\
Total & 254 & 47 & 25.4 & 207 & 55 & 17.4 \\
\hline
\end{tabular}

Tabla 3

NUMERO DE ASPIRACIONES Y EMBARAZOS CLINICOS DE ACUERDO A LA CAUSA DE INFERTILIDAD

\begin{tabular}{|lcccc|}
\hline Causas de infertilidad & No. Casos & Asp & E. Clin & \% \\
\hline Tubárica & 120 & 96 & 31 & 32.3 \\
Otras causas de infertilidad & 48 & 35 & 12 & 34.3 \\
Masculina & 19 & 13 & 2 & 15.4 \\
Múltiple & 40 & 40 & 7 & 17.5 \\
Inexplicada & 27 & 23 & 55 & 13 \\
\hline Total & 254 & 207 & & 22.5 \\
\hline
\end{tabular}


Tabla 4

EDAD VERSUS ASPIRACIONES MAS EMBARAZOS CLINICOS

\begin{tabular}{|lcccc|}
\hline Edad & No & Aspiración & E. Clínicos & $\%$ \\
\hline Menor & & & & \\
igual a 20 & 1 & 1 & 0 & 0 \\
$20-24$ & 8 & 8 & 1 & 13 \\
$25-29$ & 25 & 21 & 6 & 24 \\
$30-34$ & 85 & 78 & 21 & 25 \\
$35-39$ & 77 & 71 & 22 & 28 \\
Mayor & 58 & 29 & 5 & 8.6 \\
o igual a 40 & 254 & 208 & 55 & 16.4 \\
\hline Total & 254 & & \\
\hline
\end{tabular}

del ciclo y en un $36 \%$ de los pacientes cuando el régimen anterior se inicia al día 4 del ciclo. Las tasas de embarazo no son diferentes entre los grupos (49).

Se debe tener presente la cantidad de información que sugiere que el citrato de clomifeno puede tener efectos indeseables en el tracto reproductivo. Este antagonista de los estrógenos tiene un efecto antiestrogénico sobre el endometrio dependiendo de la dosis utilizada (50).

En vista de los posibles efectos deletéreos del citrato de clomifeno sobre el endometrio, ovocitos y la esteroidogenésis del cuerpo lúteo y a la alta incidencia de picos inoportunos de $\mathrm{LH}$, tasas de gestación relativamente bajas y tasas de aborto relativamente altas se debe preferir el uso de solo gonadotropinas para FIV (51-53). Estos efectos adversos del citrato de clomifeno no se producen cuando la medicación se ordena a donadoras de ovocitos y los ovocitos son transferidos a un endometrio receptor (54). Cuando se utilizó solo hMG en altas dosis, comenzando con 3 ampollas diarias a partir del día 3 del ciclo por 5 días seguida de la misma dosis o mayor dependiendo de la respuesta del paciente, se alcanzó un mínimo cuatro ó cinco folículos grandes, siendo posible obtener un promedio de seis ovocitos maduros con un $18 \%$ de embarazos clínicos por trasferencia y una tasa de abortos del 15\% (55-56).

La u-FSH pura fue introducida en 1980 (57), usada sola (58) o en combinación con hMG (59) seguida de la aplicación de hCG; las tasas de embarazos para diferentes combinaciones de hMG-FSH en pacientes de FIV han sido del $30 \%$ y en los que se utilizó únicamente u-FSH, la tasa de embarazos fue del $21 \%$. En un estudio extendido de más de 1300 ciclos la mayor tasa de embarazo, 25\%, se obtuvo mediante la combinación de 2 ampollas de hMG +2 ampollas de u-FSH en los días 3 ó 4 continuando con 2 ampollas de hMG según esquema establecido, la u-FSH se uso para aumentar la fase de reclutamiento (60). La tasa de éxito fue baja para el protocolo fijo de 2 ampollas diarias de hMG (21.5\%) y fue mucho más baja cuando se utilizó la dosis fija de 2 ampollas de u-FSH (17\%). Se ha sugerido que la adición de FSH a hMG al comienzo del ciclo, remeda el ciclo menstrual normal mejorando la relación
FSH/LH, manteniendo un medio ambiente intrafulicular saludable para el desarrollo del ovocito debido una disminución de la relación andrógeno - estrógeno y una baja en los niveles de testosterona (61).

Debido a las desventajas inherentes de las gonadotropinas existentes (hMG y u-FSH) como son una pureza de únicamente 1 al $2 \%$ con una alta contaminación proteica, lo cual causa reacción tisular local y dolor, siendo necesario inyectarlas intramuscularmente para evitar lo anterior. Estas preparaciones tienen una baja actividad biológica, conteniendo formas variables de gonadotropinas isoformas responsables de grandes variaciones en la vida media de estas hormonas. La vida media de la u-FSH después de aplicarla intramuscularmente varía de 30 a 40 horas, mientras que la vida media de la LH es mucho más corta y esta es la razón para administrar HCG de una vida media mayor como sustituto terapéutico del pico de la LH (62). Además, debido al origen de las preparaciones existentes estas presentan variaciones en su actividad biológica dependiendo del lote de producción. Estos problemas han hecho muy difícil preparar regímenes consistentes de formulaciones para inducción de ovulación, impidiendo que se realice un protocolo de acuerdo a las necesidades de los pacientes. Estos problemas se han solucionado utilizando la tecnología recombinante de $\mathrm{ADN}$, la cual ha conducido a la producción de r-FSH (Gonal-F, Serono Laboratorio; Puregon,Organon). La eficacia y seguridad del uso de r-FSH en la estimulación ovárica en FIV/TE fue probada en dos estudios prospectivos randomizados. En el primero (63) se comparo el uso de r-FSH (Puregon, Organon ) con u-FSH (Metrodin de Serono Laboratorios), después de haber suprimido la hipófisis con buserelina, en 981 mujeres sometidas a FIV/TE. En el grupo que recibió rFSH se obtuvo más ovocitos y embriones de mejor calidad comparado con el grupo de u-FSH. Además se necesito menos ampollas de r-FSH para estimular los ovarios comparado con u-FSH, siendo el período de tratamiento significativamente más corto, lo que indica posiblemente que esta preparación de FSH es más potente que la de origen urinario. Sin embargo, la tasa de embarazos, fue similar en los dos grupos. El segundo estudio (64) involucra el uso de Gonal -F (Serono Laboratorios) en 235 pacientes de FIV/TE (119 fueron tratados con r-FSH y 114 con uFSH (Metrodin, después de haber suprimido la hipófisis con buserelina) igualmente se obtuvo más ovocitos y embriones en el grupo en que se utilizo r-FSH, indicando que la disponibilidad de gonadotropinas con menos variabilidad entre lotes de producción podría ser benéfico.

Cuando se administran las gonadotropinas para estimular el crecimiento folicular muchos de ellos se encuentran en diferentes estados de desarrollo siendo por tanto de diferentes tamaños con una producción variable de estrógenos (64) y al ser aspirados simultáneamente, no todos están listos para ser fertilizados. Para superar esta dificultad se ha encontrado que el uso de agonistas de $\mathrm{GnRH}$ en combinación con gonadotropinas, mejora el reclutamiento y la sincronización folicular y produce una eliminación completa del pico endógeno de la LH (65-66). En los ciclos de FIV, la administración del agonista de GnRH resulta en un incremento de los ovocitos fertilizables y tasa alta de concepción por ciclo de tratamiento (67-68). 
Se ha especulado que la mejor sincronización intrafolicular se obtiene cuando el agonista de GnRh sé inicia a la mitad de la fase lútea, este efecto se obtiene por la supresión de la secreción premestrual de FSH responsable del proceso de reclutamiento folicular (67). Las ventajas del tratamiento con agonista de GnRH son limitadas por la necesidad de utilizar altas dosis de gonadotropinas para inducir ovulación y por el efecto deletéreo de esta droga en la producción de progesterona por el cuerpo lúteo. La resistencia ovárica a la administración exógena de gonadotropinas así como el efecto lúteolitico de los agonistas de la GnRH puede ser el resultado ó de la supresión pulsátil de las gonadotropinas endógenas ó un efecto inhibitorio directo del análogo sobre el ovario.

La supresión de la secreción endógena de gonadotropinas tiene dos ventajas, una la de prevenir la secreción prematura de la LH y dos extender la fase folicular, dando como resultando unos niveles altos de estradiol y en un número mayor de ovocitos recuperados.
La edad de la mujer tiene un significado estadístico en resultado de los ciclos de FIV/TE, en nuestro trabajo la tasa de embarazo clínico fue del $9 \%$ en pacientes mayores de 40 años. En la mujer, la razón principal de disminución de la fertilidad parece ser una disminución de la función ovárica y calidad de los ovocitos. Un marcador de esta declinación es la elevación de los niveles séricos de la FSH en la fase folicular temprana (día 2 ó 3 del ciclo) (69). Actualmente, una elevación de los niveles de FSH mayores de $15 \mathrm{UI} / \mathrm{L}$ en mujeres el día 3 del ciclo a cualquier edad tiene un valor predictivo de un pobre potencial reproductivo y una disminución en el número de ovocitos (70). Niveles mayores de $25 \mathrm{UI} / \mathrm{L}$ están asociadas usualmente con tasas de cero embarazos. La calidad de los ovocitos se correlaciona negativamente con la edad, particularmente para mujeres mayores de 38 años. En estas mujeres, la elevación sérica de los niveles de FSH esta asociada con tasas de implantación bajas dentro de grupo similares de edad (70).

\section{BIBLIOGRAFIA}

1. Steptoe PC, Edwards RG. Birth after reimplantation of human embryo. Lancet 1978; 2: 366-370.

2. Lopata A, Johnston JWH, Hoult IJ, Speirs AI. Pregnancy following intrauterine implantation of an embryo obtained by in vitro fertilization an embryo obtained by in vitro fertilization of preovulatory egg. Fertil Steril 1980; 33: 117-123.

3. Heape W. Preliminary note on the transplantation an growth of mammalian ova human in vitro fertilization research. Am J Obstet Gynecol 1983; 146: 477-482.

4. Kerin J, Quinn P Herriot D, Wilson L, Stone B. Effects of follicle induction on ovary and endometrium. In: De Cherney AH (Ed) The control of follicle development, ovulation and luteal function: Lessons from In Vitro Fertilizations. New York, Raven 1987; 112-124.

5. With in a uterine foster mother. Proc R Soc. 1891; 48: 457-465.

6. Hammond $\mathrm{J}$. Culture of mouse embryos using an egg-saline medium. Nature 1949; 163: 28-33.

7. Whitten WK. Culture of tubal mouse ova. Nature 1956; 177: 96-101

8. Austin CR. Observations of the penetration of the sperm into the mammalian egg. Aust J Sci Res 1951; 134: 581-586.

9. Chang MC. Fertilization capacity of spermatozoa deposited in fallopian tubes. Nature 1951; 168: 697-702.

10. Chang MC. Fertilization of rabbit ova in vitro. Nature $1959 ; 184: 406-$ 411.

11. Kering JF, Warner GM, Quinn PJ et al. Incidence of multiple pregnancy after in vitro fertilization and embryo transfer. Lancet 1983;2: 537 542.

12. Lopata A. Concepts in human in vitro fertilization and embryo transfer. Fertil Steril. 1983; 40: 289-294

13. Edwards RG. In vitro fertilization and embryo replacement: Opening lecture. Ann NY Acad. Sci. 1985; 442-449.

14. Marrs RP, Vargyas JM, Saito Het al. Clinical applications of techniques used in Smitz J, Ron- El R, Tarlatzis BC. The use of gonadotrophin releasing hormone agonist for in vitro fertilization and other assisted procreation techniques: Experiencefron three centers. Human Reprod 1992; 7(suppl) 49-54.

15. Ron-EL R, Herman A, Golan A, Nachum H, Soffer Y, Caspi E. Gonadotropins and releasing hormone agonist: Gonadotropins protocols in randomized prospective study. Fertil Steril 1991; 55: 574-579.

16. Herman A, Ron-El R, Golan A, Nachum H, Soffer Y, Caspi E. Follicle cysts following menstrual versus mid luteal administration of Gonadotropins hormone analog in IVF. Fertil Steril 1990; 53: 854-859.

17. Meldrum DR, Wiscot A, Hamilton F, Gutiay AL, Huynh D, Kempton W. Timing of initiation and dose schedule of leuprolide influence the time course of ovarian suppression. Fertil Steril 1988; 50: 400-405.

18. Ron-El R, Herman A, Golan Van der Ven H, Caspi, Diedrich K. The comparison of early follicular and mid luteal administration of long acting gonadatropin releasing hormone agonist. Fertil Steril 1990; 54: $233-238$

19. Benadiva CA, Blasco L, Tureck R, Mastroianni L, Flickinger GL. Comparison of different regimens of Gonadotropins releasing hormone analog during ovarian stimulation for in vitro fertilization. Fertil Steril 1990; 53: 479-85.

20. Lejeune B, Barlow P, Puissant F, Delvigne A, Vanrysselberge M, Leroy F. Use of busereling acetate in an in vitro fertilization program: A comparison with classical clomiphene citrate -human menopausal gonadotropin treatment. Fertil Steril 1990; 54: 475-81.

21. Abdalla HI, Ahuja KK, Leonard T, Morris NN, Honour JW, Jacobs HS. Comparative trial of lutenizing hormone realising hormone analog /human menopusal gonadotropin in an assisted conception program. Fertil Steril 1990; 53: 473-478.

22. Ferrier A, Rasweiler JJ, Bedford JM, Prey K, Berkeley AS. Evaluation of leuprolide acetate and gonadotropins for in vitro fertilization or gamete intrafallopian transfer. Fertil Steril 1990; 54: 90-95.

23. Lavy G, Restrepo Candelo H, Diamond M, Shapiro B, Grunfeld L, De Cherney AH. Laparoscopic and trasvaginal ova recovery: The effect on ova quality. Fertil Steril , 1988; 49: 1002-1006.

24. Parsons J, Riddle A, Booker M, et al. Oocyte retrieval for in vitro fertilization by ultrasonically guide needle aspiration via the urethra. Lancet 1985; 1: 1076-1081.

25. Schulman JD, Dorfmann A, Jones S, Joyce B, Hanser J: Ooccyte retrieval and local anesthesia. N Engl J Med 1985; 312: 16391644.

26. Booker M, Parsons J. The perurethral technique for ultrassound directed follicle aspiration in an in vitro fertilization and embryo transfer programme: A report of 636 patients cycles. Br J Obstet Gynecol 1990; 97: 499-504.

27. Parsons J, Pampiglione JS, Saddler AP, Booker MW, Campbell S. Ultrasound directed follicle aspiration for ooccyte collection using the perurethral technique. Fertil Steril 1990; 53: 97-102.

28. Lewin A, Laufer N, Rabinowitz R, Margalioth EJ, Bar I, Schenker JG. Ultrasonically guided oocyte collection under a local anesthesia: The first choice method for in vitro fertilization. A comparative study with laparoscopy. Fertil Steril 1986; 257-262.

29. Dellenbach P, Nisand I, Moreau, et al. Transvaginal sonographically controlled ovarian follicle punture for egg retrieval . Lancet 1984; 1: 1467-1472. 
30. Kingsland CR, Taylor CT, Aziz N, Bickerton N: Is follicular flushing necessary for oocyte retrieval ?A randomized trial . Hum Reprod 1991; 6: 382-387.

31. Coetsier T, Dhout M, De Sutter P, Merchiers E, Versichelen L, Rossell MT. Propofol anaesthesia for ultrasound guide oocyte retrieval: Accumulation of an anaesthetic agent in follicular fluid. Hum Reprod 1992; 7: 1422-1427.

32. Boyers SP, Lavy G, Rossel JB, De Cherney AH. A paired analysis of in vitro fertilization and cleavage rates, of first versus last recovered preovulatory human oocytes exposed to varying intervals of $100 \%$ pneumoperitoneum and general anesthesia. Fertil Steril 1987; 48: 969-974.

33. Schoeffler PF, Levron JC, Jany L, Brenas FJ, Pouly JL. Follicular concentration of fentanyhl during laparoscopy for oocyte retrieval: Correlation with in vitro fertilization results. Anesthesiology 1998; 69: 663-668.

34. Bailey Priedham D, Dreshef E, Drury K, Cook C L, Hurst H E, Yussman MA. Follicular fluid Lidocaine levels during trasvaginal oocyte retieval. Fertil Steril 1990; 53: 171-176.

35. Daya S, Kohut, Gamby J, Yaounglay E. Influence of blood clots in cumulus complex on oocyte fertilization and cleavage. Human Reprod 1990; 15: 744-749.

36. Veeck LL. Extracorporeal maturation. Norfolk, 1984, Ann NY Acad Sci $1985 ;$ 442: 357-367.

37. Veeck LL. The morphologic estimation of mature oocytes and their preparation for insemination. In vitro fertilization-Norfolk. Williams and Wilkins, Baltimore, 1986a; 81-92.

38. Lopata A, Patuelo MS, Chang A, James B. A method for colleting motile spermatozoa from human semen .Fertil Steril 1976; 27: 677684.

39. Mahadevan MM, Trounson AD. The influence of seminal characteristics of the succes rate of human in vitro fertilization. Fertil Steril 1984; 42: 400-405.

40. Wolf DP, Byrd W, Dandekar P, Quingley MM. Sperm concentration and fertilization of human eggs in vitro. Biol Reprod. 1984; $31: 837$ 848 .

41. Young Lai EW, Daya S. Increased fertilization of human oocyte with low density at insemination. J In Vitro Fert Embryo Transfer 1991; 8: 176-177.

42. Cohen J, De Mounzon S, Lancaster P. World Collaborative Report: VIII World Congress on In vitro Fertilization and alternate Assisted Reproduction. Oct, 1993, Kyoto, Japan.

43. AIH. National Perinatal Statistics Unit, Fertil Society of Australia: Assisted Conception Australia and New Zealand 1989, Sydney, Australia Fertility Society, 1991.

44. Sepalla M. The world collaborative report on in vitro fertilization and embryo replacement: Current State of the art in January 1984, Ann NY Acad Sci 1984; 442: 558-564.

45. Cohen J, De Mounzon J, Lancaster P, World Collaborative Report 1991, published during The 8th World Congress of IVF in Kyoto, 1993.

46. Lopata A. Concepts in human in vitro fertilization and embryo transfer. Fertil Steril 1983; 40: 289-294.

47. Mars RP, Vargas JM, Saito Het al. Clinical applications of techniques used in human in vitro fertilization research. Am J Obstet Gynecol 1983; 146: 477-482.

48. Kering J, Quinn P, Herriot D, Wilson L, Stone B. Effects of follicle induction on ovary and endometrium. In De Cherney A H (Ed) The control of follicle development, ovulation and luteal function: Lessons from In Vitro Fertilization. New York, Raven, 1987; 112-126.

49. Mahadevan MM, Fleetman J, Taylor PJ, Leader A, Pattinson AH. The effect of the day of luteinizing hormone surge and the outcome of in vitro fertilization. Fertil Steril 1987; 47: 976-981.

50. KoKKo E, Janne O, Kauppil A, Vinhko R. Cyclic clomiphene citrate treatment lowers cytosol estrogen and progentin receptor combination in the endometrium of postmenopausal women on estrogen replacement therapy. J Clin Endocrinol Metab 1981; 52: 345-536.

51. Laufer N, Pratt B, De Cherney AH, Naftolin F, Merino M, Markert CL. The in vivo in vitro effects of clomiphene citrate on ovulation, fertilization and development of coltured mouse oocytes. 1983;147: 633-638.
52. Laufer N, Barr I, Lewin A, et al. Clomiphene citrate inhibits progesterone secretion o human granulosa luteal cells in long term culture. Proceeding of the 4 th World Congress on IVF, Oct 1985, Melbourne, Australia

53. Olsson JH, Nilson L, Hillensjo T. Effect of clomiphene isomers on progestin synthesis in cultured human granulosa cells. Human Reprod 1987; 2: 463- 468.

54. Seibel MM. The effect o clomiphene citrate on human preovulatory oocyte maturation in vivo. J In Vitro Fert Embryo Transfer 1989; 6: 3-8.

55. Laufer N, De Cherney AH, Haseltine FP, et al. The use of high dose of human menopausal Gonadotropins (HMG) in an in vitro fertilization program. Fertil Steril 1983; 40: 734 -739.

56. Laufer N, De Cherney AH, Haseltine FP, et al. Human in vitro fertilization employing individualized ovulation induced by human menopausal gonadotropin. J In Vitro Fert Embryo Transfer. 1984; 1: $56-61$.

57. Jones GS, Garcia JE, Rosenwaks Z. The role of pituitary gonadotropins in follicular stimulation and ocyte maturation in the human. J. Clin Endocrinol Metab 1984; 59: 178-183.

58. Moasher SJ, Garcia JE, Rosenwaks Z. The combination of FSH and HMG for induction of multiple follicular maturation for IVF. Ferti Steril 1985; 44: 62-68.

59. Jones GS, Acosta AA, Garcia JE, Bernardus RE, Rosenwaks Z. The effect of FSH without additional LH, on follicular stimulation and oocyte development in normal ovulatory women. Fertil Steril 1985; 43: 696-701

60. Navot D, Rosenwaks Z. The use of FSH for controlled ovarian hyperstimulation in IVF. J. In Vitro Fert Embryo Transfer 1988; 5: 3-8.

61. Polan ML, Danielle A, Russel JB. Ovulation induction with HMG compared to pure FSH results in differences in FF androgen levels. Presented at the 42 nd Annual Meeting of the American Fertil Society, April, 1986, Toronto, Ontario

62. Mizununa H, Takagi T, Honjyo S, Ibuki Y, Igarashi M. Clinical pharmacodynamics of urinary follicle stimulating hormone and its applications for pharmacokinetic stimulation program. Fertil Steril 1990; 193: 263-271

63. Out HJ, Mannaerts BMJL, Driessen SGAJ, Coelingh-Bennink HJT. A prospective, randomized, multicentre study comparing recombinant and urinary follicle stimulating hormone (Puregon vs. Metrodin) in vitro fertilization. Human Reprod 1995; 10: 2534-2540.

64. Laumaye E, Alvarez S, Barlow D et al. Efficacy of recombinant human follicle stimulanting hormone (GonalF) for stimulating multiple follicular development in assisted reproduction technologies In Morit , Aono T, Tominaga T, Hiroi M (eds) Proceeding s of the VIII Th World Congress on in vitro Fertilization and Alternate Assisted Reproduction, Kioto, Japan, Sep 1993.

65. Flemening R, Haxtron MJ, Hamilton MP et al. Successful treatment of infertil women with oligomenorrea using a combination of an LH-RH agonist and exogenous gonadotropins. Br J Obstet Gynaecol 1985; 92: 369-375.

66. Neven S, Hedon B, Bringer J et al. Ovarian stimulation by combination of a gonadotropin-realizing hormone agonist and Gonadotropins for in vitro fertilization Fertil Steril 1987; 47: 639-645.

67. Palermo R, Amadeo G, Navot D, Rosenwacks R. Concomitant gonadotropin-realizing hormone agonist and menotropin treatment for the synchronized induction of multiple follicles. Fertil Steril 1988; 48: $290-296$

68. Smitz J, Ron-El R, Tarlatzis BC. The use of gonadotropin realizing hormone agonist for in vitro fertilization and other assisted procreation techniques: Experience from the three center. Human Reprod 1992; (Suppl 1) 7: 49-55

69. Lenton EA, Sexton L, Lee S, Cooke ID. Progressive Changes in LH/ FSH ratio in women throughout reproductive live. Maturitas, 1988; 10: 35-39.

70. Scott RD, Toner JP, Muasher SJ. Follicle-stimulanting hormone levels on cycle day 3 are predictive of in vitro fertilization outcome. Fertil Steril 1989; 51 651-656. 